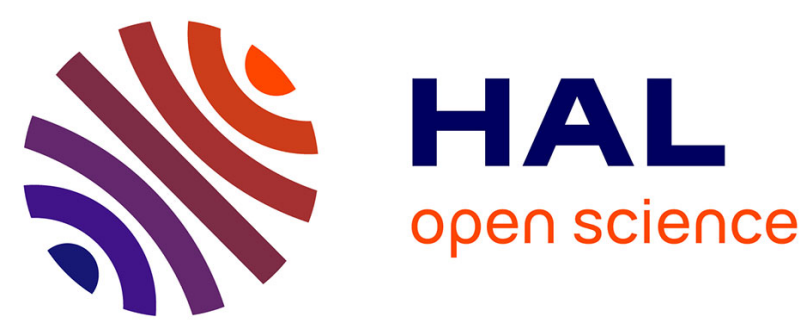

\title{
The electronic properties of mixed valence hydrated europium chloride thin film
}

\author{
M.G. Silly, Fabrice Charra, François Lux, G. Lemercier, F. Sirotti
}

\section{To cite this version:}

M.G. Silly, Fabrice Charra, François Lux, G. Lemercier, F. Sirotti. The electronic properties of mixed valence hydrated europium chloride thin film. Physical Chemistry Chemical Physics, 2015, 17, pp.18403. 10.1039/c5cp01256b . cea-01349746

\section{HAL Id: cea-01349746 https://hal-cea.archives-ouvertes.fr/cea-01349746}

Submitted on 2 Oct 2017

HAL is a multi-disciplinary open access archive for the deposit and dissemination of scientific research documents, whether they are published or not. The documents may come from teaching and research institutions in France or abroad, or from public or private research centers.
L'archive ouverte pluridisciplinaire HAL, est destinée au dépôt et à la diffusion de documents scientifiques de niveau recherche, publiés ou non, émanant des établissements d'enseignement et de recherche français ou étrangers, des laboratoires publics ou privés. 


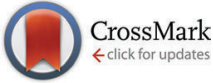

Cite this: Phys. Chem. Chem. Phys., 2015, 17, 18403

Received 3rd March 2015, Accepted 16th June 2015

DOI: $10.1039 / c 5 c p 01256 b$

www.rsc.org/pccp

\title{
The electronic properties of mixed valence hydrated europium chloride thin film
}

\author{
M. G. Silly, ${ }^{\text {a }}$ F. Charra, ${ }^{b}$ F. Lux, ${ }^{c}$ G. Lemercier ${ }^{d}$ and F. Sirotti ${ }^{a}$
}

We investigate the electronic properties of a model mixed-valence hydrated chloride europium salt by means of high resolution photoemission spectroscopy (HRPES) and resonant photoemission spectroscopy (RESPES) at the Eu $3 d \rightarrow 4 f$ and $4 d \rightarrow 4 f$ transitions. From the HRPES spectra, we have determined that the two europium oxidation states are homogeneously distributed in the bulk and that the hydrated salt film is exempt from surface mixed valence transition. From the RESPES spectra, the well separated resonant contributions characteristic of divalent and trivalent europium species $\left(4 f^{6}\right.$ and $4 f^{7}$ final states, respectively) are accurately extracted and quantitatively determined from the resonant features measured at the two edges. The partial absorption yield spectra, obtained by integrating the photoemission intensity in the valence-band region, can be well reproduced by atomic multiplet calculation at the $M_{4,5}(3 d-4 f)$ absorption edge and by an asymmetric Fano-like shape profile at the $\mathrm{N}_{4,5}(4 \mathrm{~d}-4 \mathrm{f})$ absorption edge. The ratio of $\mathrm{Eu}^{2+}$ and $\mathrm{Eu}^{3+}$ species measured at the two absorption edges matches with the composition of the mixed valence europium salt as determined chemically. We have demonstrated that the observed spectroscopic features of the mixed valence salt are attributed to the mixed-valence ground state rather than surface valence transition. HRPES and RESPES spectra provide reference spectra for the study of europium salts and their derivatives.

\section{Introduction}

Due to their optical properties, rare-earth ions are of high interest for applications in photoactive organometallic compounds such as light-emitting devices (OLEDs, phosphors, etc.), chemical and biological sensors, and wavelength photoconverters for photovoltaic applications. ${ }^{1}$ More recently rare-earth ions in dielectric crystals have also shown high potential in quantum information processing as optical quantum memories or as quantum repeaters in quantum communication devices..$^{2-6}$ Nevertheless, despite the $4 \mathrm{f}$ core shielding by the surrounding filled $5 \mathrm{~s}$ and $5 \mathrm{p}$ shells, luminescent ${ }^{7}$ and electronic ${ }^{6}$ properties of Eu ions suffer from their sensitivity to the chemical environment such as counter ions, water and solvents, at the origin of inhomogeneous broadening on optical transitions ${ }^{6}$ limiting the bandwidth and the efficiency of quantum memory based on electromagnetically induced transparency. ${ }^{8-10}$ Moreover, Eu luminescence emission

\footnotetext{
${ }^{a}$ TEMPO Beamline, Synchrotron SOLEIL, St. Aubin, BP 48, 91192 Gif-sur-Yvette Cedex, France. E-mail: mathieu.silly@synchrotron-soleil.fr

${ }^{b}$ Service de Physique de l'Etat Condensé, CEA-CNRS UMR 3680,

Institut Rayonnement Matière de Saclay (IRAMIS), F-91191 Gif-sur-Yvette Cedex, France

${ }^{c}$ Institut Lumière Matière - UMR 5306 - Bât Jules Raulin; 22,

avenue Gaston Berger - Domaine de la Doua, 69622 Villeurbanne Cedex, France

${ }^{d}$ Université Reims Champagne-Ardenne, ICMR UMR CNRS no. 7312, Groupe

Chimie de Coordination, BP 1039, 51687 Reims Cedex 2, France
}

strongly depends on $4 \mathrm{f}$ states: while $\mathrm{Eu}^{3+}$ presents a pure atomic-line-like red luminescence, $\mathrm{Eu}^{2+}$ exhibits a strong UV broad band luminescence. ${ }^{11}$

Recently a stable phase in UHV of a hydrated $\mathrm{EuCl}_{2.85}$ salt has been evidenced. ${ }^{12}$ In general mixed-valence compounds present high interest in electronic applications as fast ion conductors but also mixed ionic-electronic conductors. ${ }^{13}$ They notably find applications as solid electrolytes or electrodes in energy storage domains as substituents to liquid electrolytes. The specific conducting properties are governed by defects and they depend directly on the amount, arrangement and localization of different ions in the bulk. It has been demonstrated that the conductivity of europium oxide significantly varies with its stoichiometry ${ }^{14}$ and can be tuned to match that of silicon. ${ }^{15,16}$ But the amount and the location of oxygen vacancies at the origin of doping are still difficult to assess. ${ }^{17}$

An accurate determination of the composition of the salt combined with a better understanding of the spatial distribution into the ionic solid of different oxidation states of europium is susceptible to provide insights into the expected conductivity of the salt. It has been shown that in trivalent rare-earth (RE) compounds, surface valence transitions to the divalent state accompany the change in coordination at the surface which favors the lower-valence state of the RE ions. ${ }^{18,19}$ This process manifests itself as a surface core level shift in photoemission spectroscopy experiments. ${ }^{20,21}$ Although trivalent Eu contribution 
measured in photoemission was for a while explained by shakeup excitations in insulating RE compounds, ${ }^{22}$ this explanation has been finally refuted in favor of the surface valence transition. ${ }^{21}$ The origin of divalent europium can be unambiguously identified by measuring the core level spectra at different photon energies and taking into account the different electron escape depth $\lambda$ depending on the photoelectron kinetic energy. ${ }^{23,24}$ If the $\mathrm{Eu}^{2+}$ species are located at the surface, the electrical conduction will come from the domain boundary, whereas if they are homogeneously distributed, the charges will lead to bulk conduction. The deviation from stoichiometry of a pure hydrated $\mathrm{EuCl}_{3}$ salt induces structural disorder and electronic compensation leading to the creation of electronic defects, here, lower oxidation states $\left(\mathrm{Eu}^{2+}\right)$ at the origin of electrical conductivity. An excess of metallic ions leads to $n$ doped semiconductors. Whereas many studies have been conducted on metal oxides and alloys, few ones have focused on europium salts. The signature of the electronic structure and potential interactions with ligands in europium salt are missing. A better understanding of these interactions, which often limit the device performances, is needed in view of the development of applications.

Independent of high resolution core level photoemission spectroscopy (HRPES), near-edge X-ray absorption fine structure (NEXAFS) and resonant photoemission spectroscopy (RESPES) techniques according to their high chemical sensitivity have been used, mainly to study the composition of mixed valence compounds. Due to the large $4 \mathrm{f}$ photoemission enhancement observed at the $3 \mathrm{~d}, 4 \mathrm{~d} \rightarrow 4 \mathrm{f}$ resonance, absorption and resonant photoemission spectroscopies represent powerful tools to study valence changes in rare-earth ions in dilute systems and at surfaces. ${ }^{25}$ These techniques have been successfully employed to determine oxidation states in various mixed valence compounds including europium based metallic oxides, ${ }^{26}$ europium intermetallic compounds, ${ }^{27,28}$ and magnetic compounds, ${ }^{29,30}$ nevertheless surface effects such as surface valence changes in intermetallic compounds underline the extreme care needed to interpret the measured signals. ${ }^{31}$ Actually, in the solid state, the complexity and the increased number of different recombination processes contributing to features observed in resonant photoemission experiments can lead to supplementary information about e.g. charge transfer processes. ${ }^{32,33}$ Usually, the NEXAFS experiment needs a spectroscopic reference to properly assign the electronic properties and chemical composition of a material. ${ }^{12,31,34}$ Here it is shown that the use of the combination of HRPES, NEXAFS and RESPES overcomes this limitation and provides a powerful method to determine both the composition as well as the distribution of various oxides at the surface. ${ }^{29}$

In this paper, we report a detailed study on high-resolution $4 \mathrm{~d}$ photoelectron spectra and resonant soft X-ray $3 \mathrm{~d} \rightarrow 4 \mathrm{f}$ and $4 \mathrm{~d} \rightarrow 4 \mathrm{f}$ excitations of a hydrated mixed valence europium salt. We provide a reference of the electronic structure of the $\mathrm{EuCl}_{2.85}$ salt composed of a mixture of $\mathrm{Eu}^{2+}$ and $\mathrm{Eu}^{3+}$ ions. From different spectroscopic techniques, we extract the signature of each hydrated europium chloride salt valence. By comparison with theoretical models describing the X-ray absorption spectra of pure valence species, we have determined the contribution of each species, quantified them and have confirmed the homogeneous repartition of the oxides in the salt. We have shown the accuracy and the complementarity of HRPES and RESPES to characterize the electronic properties of complex mixed valence compounds such as europium based ionic solids.

\section{Experimental setup}

Hydrated europium chloride thin films were formed on the gold substrate by depositing a commercial $\mathrm{EuCl}_{3} \cdot 6 \mathrm{H}_{2} \mathrm{O}$ salt (Acros) with a purity of $99.9 \%$. Samples were prepared by dissolving the $\mathrm{EuCl}_{3} \cdot 6 \mathrm{H}_{2} \mathrm{O}$ salt in dry ethanol. The solution was then deposited on the gold substrate, and solvent was evaporated at $50{ }^{\circ} \mathrm{C}$ under a $\mathrm{N}_{2}$ gas flux. Gold substrates were $100 \mathrm{~nm}$ thick layers of gold epitaxially grown on mica. Before use, they were $\mathrm{H}_{2}$ flame annealed at about $450{ }^{\circ} \mathrm{C}$ in air in order to obtain clean $\mathrm{Au}(111)-22 \times \sqrt{ } 3$ reconstructed terraces.

A recent study ${ }^{12}$ based on a combination of chemical dosing, core level photoemission spectroscopy, X-ray absorption, and photoluminescence showed that the europium salt prepared following this procedure and placed in an UHV environment presents a homogeneous assembly of hydrated $\mathrm{EuCl}_{2}$ and $\mathrm{EuCl}_{3}$ species. Chemical dosing by ionic chromatography leads to a $\mathrm{EuCl}_{2.85 \pm 0.05}$ composition in good agreement, within measurement accuracy, with electronic properties exhibiting a contribution of $15 \%<\mathrm{Eu}^{2+}<20 \%$ and $80 \%<\mathrm{Eu}^{3+}<85 \%$ species.

Eu 4d HRPES, X-ray absorption spectroscopy (XAS) and RESPES experiments at $\mathrm{Eu} 3 \mathrm{~d} \rightarrow 4 \mathrm{f}$ and $4 \mathrm{~d} \rightarrow 4 \mathrm{f}$ absorption edges were performed at the French Synchrotron Facility Soleil at the soft X-ray TEMPO beamline. ${ }^{35}$ The XAS was performed by recording the drain sample current as a function of photon energy and normalized to a gold mesh signal (60\% transmission). HRPES and RESPES experiments were performed using a hemispherical electron analyser Scienta 2002 equipped with a fast delay line detector. ${ }^{36}$ The total resolving power of the experiments (beamline and electron energy analyser resolving power) was better than 5000. Measurements were performed at a pressure lower than $3 \times 10^{-10} \mathrm{mbar}$. To analyze europium ion absorption spectra measured at $3 \mathrm{~d} \rightarrow 4 \mathrm{f}$ and $4 \mathrm{~d} \rightarrow 4 \mathrm{f}$ transitions, atomic multiplet calculations were performed using the CTM4XAS 5.23 program $^{37}$ based on Cowan atomic calculation codes. ${ }^{38}$

The inelastic electron mean free paths for the hydrated $\mathrm{EuCl}_{3} \cdot 6 \mathrm{H}_{2} \mathrm{O}$ salt were calculated using the QUASES-IMFP-TPP2M software based on the Tanuma Powell and Penn algorithm (TPP2M). ${ }^{39}$ A bulk density of $4.89 \mathrm{~g} \mathrm{~cm}^{-3}$ and a molecular weight of $366.414 \mathrm{u}$ are used according to the literature. ${ }^{40}$

Resonant photoemission experiments were performed by scanning the photon energy of an impinging soft- $\mathrm{X}$ ray beam across the threshold energy needed to excite an electronic transition from a core level to allowed empty states. In the case of europium we have studied $3 \mathrm{~d} \rightarrow 4 \mathrm{f}$ and $4 \mathrm{~d} \rightarrow 4 \mathrm{f}$ electronic excitations located at around $1130 \mathrm{eV}$ and $150 \mathrm{eV}$, respectively. The process is schematized in Fig. 1. In the X-ray absorption 


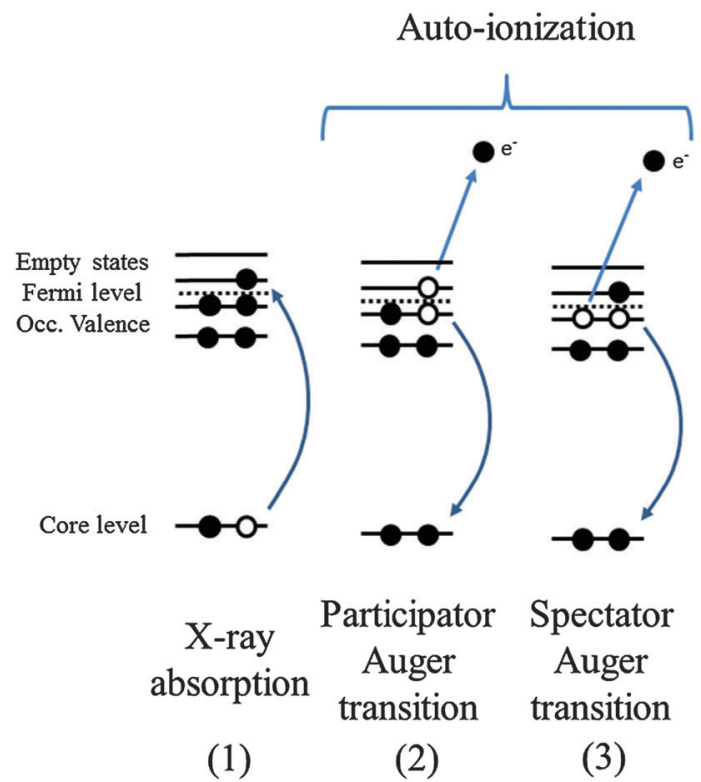

Fig. 1 Schematic of an electronic orbital diagram describing the resonant photoemission excitation and de-excitation processes. (1) Resonant X-ray absorption, exciting photon energy corresponds to a transition from an occupied core level to a valence band empty state. (2) and (3) Deexcitation processes accompanied by emission of electrons referred to as participator (2) and spectator (3) Auger transitions, respectively.

process a core-electron is photo-excited into a resonant bound state [Fig. 1-(1)]. The system can then relax via Auger and fluorescence transitions involving all external electronic states. Two types of Auger processes are possible in the relaxation process. In the participator-type Auger transition the photoexcited electron itself is involved in the Auger emission process [Fig. 1] and disperses with a constant binding energy [Fig. 1-(2)], while the spectator Auger and normal Auger disperse with constant kinetic energy when the photon energy is scanned through the absorption edge [Fig. 1-(3)].

We measured valence-band resonant photoemission spectra by analyzing in a binding energy range of about $20 \mathrm{eV}$ below $E_{\mathrm{F}}$ and observed the de-excitation processes in which valence electrons are directly involved. The outcome of the experiment is a three dimensional map where the photoemission intensity is presented as a function of impinging photon energy and binding energy as deduced from photo-electron kinetic energy. Further details of the techniques can be found elsewhere. ${ }^{30,41}$

During the resonant photoemission experiment, the total electron yield (TEY) emitted can be measured as a function of the photon energy by detecting the overall drain current. The $\mathrm{X}$-ray absorption spectrum can be directly superimposed onto the resonant photoemission map. $\mathrm{Eu}^{2+}$ and $\mathrm{Eu}^{3+}$ absorption spectra were simulated using calculations based on the atomic multiplet theory for divalent and trivalent europium at the $3 \mathrm{~d} \rightarrow$ 4f absorption edge using the CTM4XAS 5.23 program. ${ }^{37}$ The discrete energy states are broadened with a Gaussian and a Lorentzian shape to take into account the enlargement of discrete energy states due to instrumental resolution and core-hole lifetime, respectively.

\section{Results and discussion}

\subsection{Core level photoemission}

In Fig. 2, we report the Eu $4 \mathrm{~d}$ core level photoemission spectra, presenting the final state of the hydrated mixed valence compound salt, measured at two photon energies.

By comparing the $4 \mathrm{~d}$ core level spectra measured for two different photon energies, Fig. 2a, a depth profile analysis is
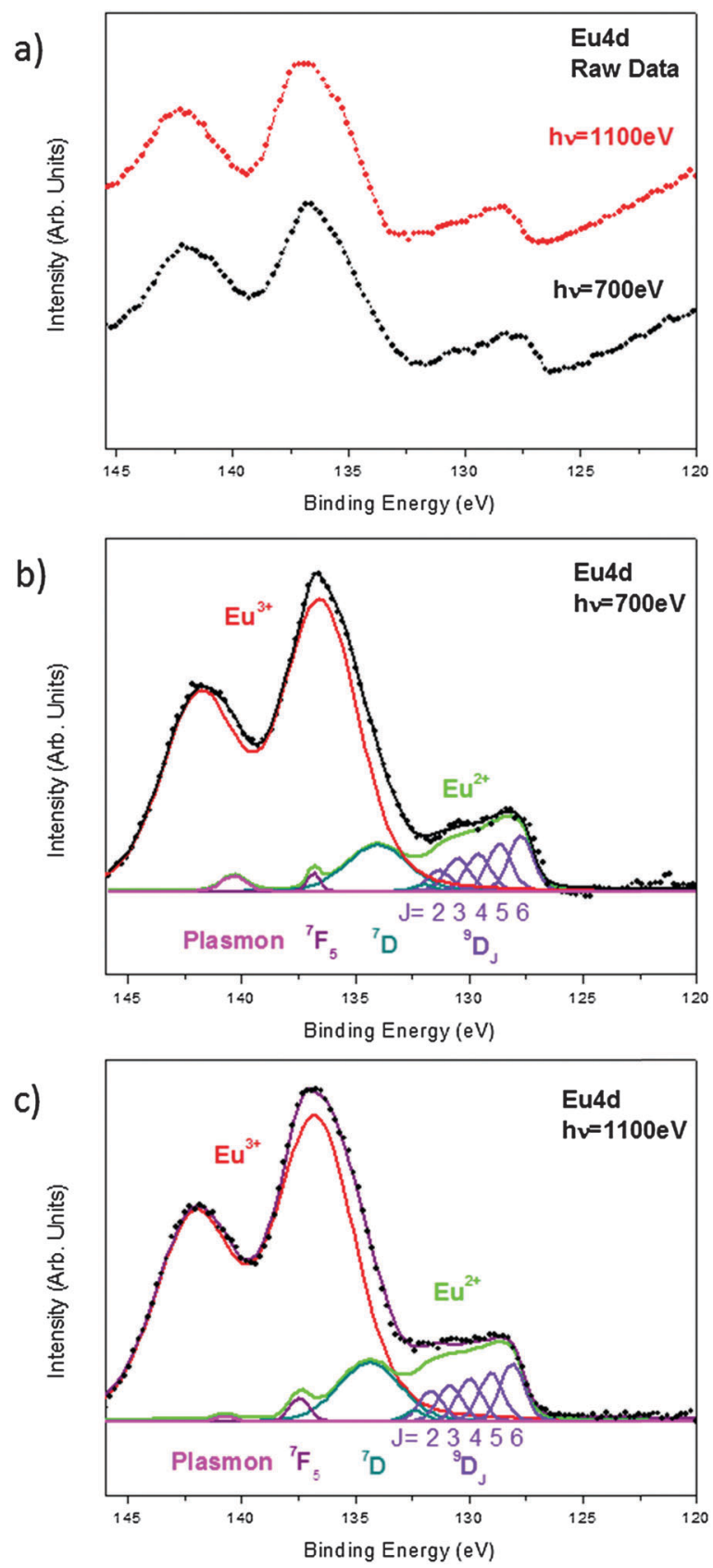

Fig. 2 (a) High resolution Eu 4d photoelectron spectra of the hydrated $\mathrm{EuCl}_{2.85}$ mixed valence salt measured at $700 \mathrm{eV}$ and $1100 \mathrm{eV}$ to perform a depth profile analysis. The results of curve fitting for the spectra measured at $700 \mathrm{eV}$ (b) and $1100 \mathrm{eV}$ (c) are presented. The divalent feature exhibits a complex shape caused by $4 d-4 f$ interactions, the signal is deconvoluted according to ref. 42 . 
performed to extract the distribution of the divalent europium in the thin film. Here we show that the shape of the $4 \mathrm{~d}$ core level spectra does not exhibit noticeable modification for photon energies of $700 \mathrm{eV}$ and $1100 \mathrm{eV}$ corresponding to $570 \mathrm{eV}$ and $970 \mathrm{eV}$ in kinetic energy, respectively. The two kinetic energies correspond to an electron inelastic mean free path $(\lambda)$ of $13.14 \AA$ and $19.38 \AA$, respectively. At normal emission, the probability of electron escape depth $\phi(z)$ follows a Beer-Lambert law:

$$
\phi(z) \propto \exp (-z / \lambda)
$$

Integrating the equation with respect to the depth $(z)$, it appears that $95 \%$ of the photoemission signal is originated in the first $3 \lambda$ in thickness. The measured core level spectra are very sensitive to the signal originated from the surface. To quantitatively determine the variation of the $\mathrm{Eu}^{2+}$ and $\mathrm{Eu}^{3+}$ amount as a function of photon energy, we have performed a fine decomposition of the core level spectra. In Fig. $2 \mathrm{~b}$ and c, the Eu $4 \mathrm{~d}$ core level spectra, which are measured at $700 \mathrm{eV}$ and $1100 \mathrm{eV}$ in photon energy, respectively, are deconvoluted using Voigt functions. Components located at lower binding energy correspond to $\mathrm{Eu}^{3+}$ states, a standard Voigt doublet taken into account for the spin orbit splitting of $5.3 \mathrm{eV}$ and a ratio of $2: 3$ for the $4 \mathrm{~d}_{3 / 2}$ and $4 \mathrm{~d}_{5 / 2}$ states, respectively. To match with the experimental data a Gaussian broadening of $1.5 \mathrm{eV}$ is used. The remaining signal located at higher kinetic energy related to the $\mathrm{Eu}^{2+}$ states exhibits a more complex shape due to multiplet interactions. The $\mathrm{Eu}^{2+} 4 \mathrm{~d}$ core level spectra exhibit the characteristic multiplet structure induced by a strong interaction between the $4 \mathrm{~d}$ hole and the unfilled $4 \mathrm{f}$ shell, as the two electron shells have the same principal quantum number. The $\mathrm{Eu}^{2+} 4 \mathrm{~d}$ core level is deconvoluted according to the experimental and theoretical work on atomic europium. ${ }^{42}$ The coupling between the d-hole and the ground state configuration of Eu leads mainly to ${ }^{7} \mathrm{D}_{J}(J=1$ to 5) states and ${ }^{9} \mathrm{D}_{J}(J=2$ to 6$)$ states, but two other contributions attributed to the plasmon and the ${ }^{7} F_{5}$ state are also noticeable at higher binding energy. Compared to the energy position found in atomic europium, ${ }^{42}$ the binding energy positions in the salt are shifted by $8.2 \mathrm{eV}$ in lower binding energy. Indeed, the values in the gas phase have to be pondered by the work function of the solid and the cohesive energy varying according to the europium environment and chemical bonding. ${ }^{43}$ The binding energy positions we find for the Eu salt are close to that found in europium based semiconductors, ${ }^{29,44}$ and europium intermetallic $^{20}$ and insulating compounds. ${ }^{21,22}$ For the deconvolution of ${ }^{9} \mathrm{D}$ states, we use the natural line width of $0.2 \mathrm{eV},{ }^{42}$ to match the data, we determine a Gaussian broadening of $0.7 \mathrm{eV}$ higher than the instrumental resolution. The FWHM of ${ }^{9} \mathrm{D}$ transitions gives a direct insight into the disorder, inhomogeneity and defects in the sample and of the electronic interactions with surrounding ligands in the crystal, as both of them result in a broadening of different components. Compared to the literature, the FWHM appears to be larger than that one observed in atomic europium ${ }^{42}$ or in well defined crystals such as $\mathrm{Eu}_{1-x} \mathrm{Gd}_{x}$ Te semiconductors ${ }^{29}$ but is comparable to that one measured for insulating $\mathrm{Eu}$ compounds presenting a mixed valence transition. ${ }^{21,22}$ Mainly two phenomena contribute to the observed broadening, the high sensitivity of the europium ion to the molecular environment and notably the interaction with surrounding ligands $\left(\mathrm{Cl}^{-}, \mathrm{H}_{2} \mathrm{O}\right)$ enlarging the optical transition as observed in $\mathrm{THz}$ experiments ${ }^{6}$ and the coexistence of $\mathrm{Eu}^{3+}$ and $\mathrm{Eu}^{2+}$ oxidation states in the salt introducing $\mathrm{Cl}^{-}$vacancies into the crystal assembly. In comparison, we have seen that the $\mathrm{Eu}^{3+} 4 \mathrm{~d}$ component presents a Gaussian broadening of $1.5 \mathrm{eV}$ higher than the broadening for $\mathrm{Eu}^{2+9} \mathrm{D}$ states. As an ionic crystal is governed by Coulomb interaction between the ions, the $\mathrm{Eu}^{3+}$ oxide also presents a high sensitivity to the chemical environment and is affected by the crystal defect induced by $\mathrm{Eu}^{2+}$.

From the contribution coming from the divalent and trivalent europium, we calculate the $\mathrm{Eu}^{2+}: \mathrm{Eu}^{3+}$ ratio for the two photon energies (700 eV and $1100 \mathrm{eV})$. In spite of the peak fitting uncertainty due to a close imbrication of the divalent and trivalent europium signals, we find, in both cases, a $\mathrm{Eu}^{2+}: \mathrm{Eu}^{3+}$ ratio of $2: 8$ corresponding to $20 \%$ of divalent europium. As the $\mathrm{Eu}^{2+}: \mathrm{Eu}^{3+}$ ratio remains constant as a function of the probing photon energy, we can conclude that no surface processes are responsible for the observed $\mathrm{Eu}^{2+}$ contribution. This result argues in favor of a homogeneous repartition of the divalent europium in the salt. The determined $\mathrm{Eu}^{2+}: \mathrm{Eu}^{3+}$ ratio, although slightly higher than the expected ratio of $15 \%$ of divalent europium determined by chemical dosing, remains within the error bar. ${ }^{12}$

\subsection{Resonant photoemission signal}

The resonant photoemission signal of the europium ion is measured at the $4 \mathrm{~d}$ and $3 \mathrm{~d}$ absorption edges where the spectroscopic signals coming from the two oxidation states are well separated. ${ }^{26-33}$

The results obtained at the $\mathrm{Eu} 3 \mathrm{~d} \rightarrow 4 \mathrm{f}$ transition $\left(\mathrm{M}_{4,5}\right.$ absorption edge) are reported in Fig. 3. In Fig. 3a, the black curve represents the XAS spectrum measured in total electron yield in the photon energy range of 1118-1178 eV. Two main contributions located at 1131 and $1158.9 \mathrm{eV}$ are visible and correspond to Eu $3 \mathrm{~d}_{5 / 2} \rightarrow 4 \mathrm{f}$ and $3 \mathrm{~d}_{3 / 2} \rightarrow 4 \mathrm{f}$ transitions, respectively. The observed features are consistent with a signal dominated by $\mathrm{Eu}^{3+}$ species ${ }^{45,46}$ but the slight shoulder located at $1128.8 \mathrm{eV}$ (indicated by a black stick in the top panel in Fig. 3a) denotes a noticeable contribution of $\mathrm{Eu}^{2+}$. The detailed shape is in good agreement with an atomic multiplet structure of a mixture of $\mathrm{Eu}^{2+} / \mathrm{Eu}^{3+}$ species. $^{27}$

The origin of the structures corresponding to different oxidation states identified in the X-ray absorption spectrum can be better disentangled by observing the resonant photoelectron spectroscopy map measured in the binding energy range between the Fermi level and $25 \mathrm{eV}$ as reported in Fig. 3b. The photoemission intensity is presented in a pseudo-color scale as a function of the photon energy ( $x$ axis) and of the electron binding energy ( $y$ axis). Due to the large photoionization cross section at the $3 \mathrm{~d} \rightarrow 4 \mathrm{f}$ transition, resonant photoemission is very sensitive to the europium species. The resonant signals coming from various oxidation states exhibit sharp and well-separated structures. The oxidation states present in the sample can be unambiguously ascribed and accurately quantified. Looking at the resonant intensity we can discern the contributions from 


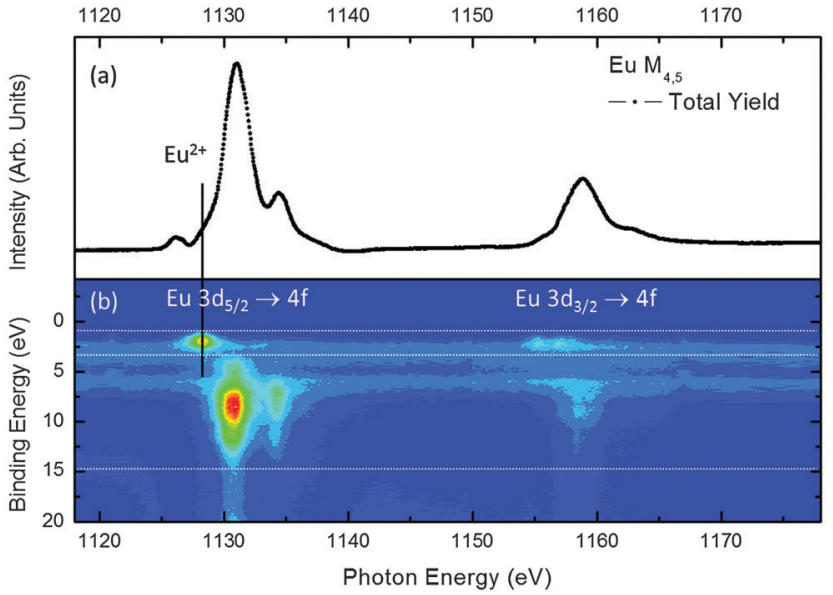

Fig. 3 (a) Near band edge $\mathrm{X}$-ray absorption spectrum for the mixed valence $\mathrm{EuCl}_{2.85}$ salt deposited on the $\mathrm{Au}(111)$ reconstructed surface measured in the photon energy range of Eu $3 d-4 f\left(M_{4,5}\right.$ absorption edge) in TEY mode. (b) Resonant photoemission map of the photon energy (1118-1178 eV) corresponding to $3 d \rightarrow 4 f$ absorption edges. The right part corresponds to $3 d_{3 / 2} \rightarrow 4 f$ transitions. The left part is related to $3 d_{5 / 2} \rightarrow 4 f$ transitions, dash lines delimit the area of localized resonant states.

two resonant Auger decay channels localized at two welldefined energy regions within the binding energy scale. When gradually increasing the photon energy, in the Eu $3 d_{3 / 2} \rightarrow 4 f$ transitions, we first identify a sharp single resonance located at $2.2 \mathrm{eV}$ of binding energy. In the literature, this feature is commonly attributed to $\mathrm{Eu}^{2+}$ states. ${ }^{27,47}$ It is followed by a broader resonant peak located between 3 and $12 \mathrm{eV}$ with a maximum close to $9 \mathrm{eV}$. This second structure is interpreted in terms of $\mathrm{Eu}^{3+}$ resonances. ${ }^{27}$ The same behavior is observed at the Eu $3 \mathrm{~d}_{5 / 2} \rightarrow 4 \mathrm{f}$ transition at about $1160 \mathrm{eV}$ where a resonant state located at $2.25 \mathrm{eV}$ and a broad resonance centered at $7 \mathrm{eV}$ are also ascribed to $\mathrm{Eu}^{2+}$ and $\mathrm{Eu}^{3+}$ states, respectively.

The resonant components shift linearly with the photon energy and are in good agreement with participator-type Auger transition models. The observed resonant de-excitation pathways involving discrete $3 \mathrm{~d} \rightarrow 4 \mathrm{f}$ transitions lead to the emission of $4 \mathrm{f}$ valence electrons of well-defined binding energy. From the intensity map one can extract resonant absorption line profiles by isolating each resonant state in its own well-defined integration domain of binding energies (partial yield). The integrated profiles of the resonant photoemission lines located at binding energies of $2.26 \mathrm{eV}$ and $7 \mathrm{eV}$ (Fig. 3) are reported in the top panel of Fig. 4a. In order to unambiguously ascribe resonant features to different europium species we compare the partial yield resonant profile to the results of atomic multiplet calculations. The calculated Eu $3 \mathrm{~d} \rightarrow 4$ f XAS spectra of $\mathrm{Eu}^{2+}$ and $\mathrm{Eu}^{3+}$ species are reported in the central panel of Fig. $4 \mathrm{~b}$. They well reproduce the partial yield spectra reported in the top panel of Fig. 4a. Discrete energy states whose broadening leads to the absorption spectrum are presented in the bottom panel of Fig. 4c. The best match with the experimental data was obtained with a Gaussian half-width half-maximum of $0.6 \mathrm{eV}$ representing the experimental broadening and a Lorentzian half-width halfmaximum of $0.2 \mathrm{eV}$ in good agreement with the core-hole natural width used in a theoretical study. ${ }^{45}$ The main features present in each valence of europium are well reproduced: the main peaks at $1128.3 \mathrm{eV}$ and the double structure at 1131 and $1134.4 \mathrm{eV}$ can be ascribed to the divalent and trivalent ions, respectively. The energy position of $\mathrm{Eu}^{2+}$ contribution fits with the shoulder at $1128.8 \mathrm{eV}$.

Nevertheless, some differences in the partial yield spectra must be underlined. The small peak at $1126.2 \mathrm{eV}$ photon energy in the trivalent $\mathrm{Eu}$ ion is not observed in the partial yield spectra but is present in the total electron yield spectrum of Fig. 4. This means that the decay process following this absorption is not accompanied by the emission of electron coming from the valence band region. The relative intensity of $\mathbf{M}_{4}$ and $\mathbf{M}_{5}$ absorption edges measured in TEY is not the same observed in the MVV partial yield spectra. The $\mathbf{M}_{4}$ structure measured in partial yield must be multiplied by a factor of 3.1 to reach the same spectral intensity obtained in total electron yield mode. This is due to a $M_{4} M_{5} V$ Coster Kronig decay in the Eu $\mathbf{M}_{4}$ excited state of the same order of the one observed on transition metals. ${ }^{48}$ The good match of the $\mathrm{Eu}^{2+}$ and $\mathrm{Eu}^{3+}$ calculated multiplet spectral structure to integrated resonant photoemission lines located at binding energies of $2.26 \mathrm{eV}$ and $7 \mathrm{eV}$ permits us to unambiguously assign the two measured resonant features to the two different

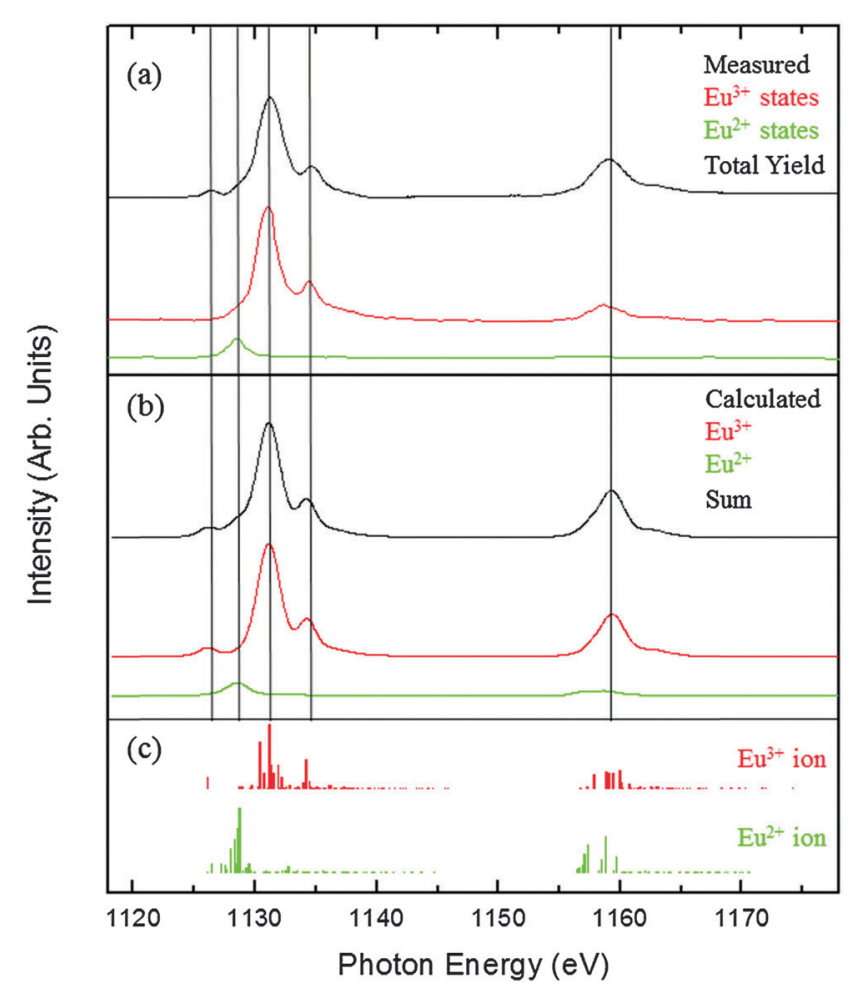

Fig. 4 (a) $M_{4,5}$ absorption edges (black line) measured in TEY. Red and green lines are obtained by integrating the resonant valence band photoemission intensity around the two main structures situated at $2.26 \mathrm{eV}$ and $7 \mathrm{eV}$ binding energies, respectively. (b) Europium $M_{4,5}$ absorption spectra calculated using an atomic multiplet model using the CTM4XAS 5.23 program. The $\mathrm{Eu}^{3+}$ and $\mathrm{Eu}^{2+}$ valences are presented in red and green whereas we present in black the sum of the two with a relative weight of 15 and $85 \%$ respectively. (c) Sticks corresponding to discrete energy states of intraatomic multiplet excitation of $\mathrm{Eu}^{2+}$ and $\mathrm{Eu}^{3+}$ ions. 
oxidation states. We can conclude that resonant photoemission processes at $\mathbf{M}_{4,5}$ absorption edges are explained in sole terms of discrete intra-atomic multiplet transitions broadened by core-hole lifetime and instrumental resolution.

The resonant signals can consequently be explained in terms of two step excitation/de-excitation processes verifying the following transitions:

$$
\text { Eu } 3 \mathrm{~d}^{10} 4 \mathrm{f}^{n}+h \nu \rightarrow\left(\operatorname{Eu~} 3 \mathrm{~d}^{9} 4 \mathrm{f}^{n+1}\right)^{*} \rightarrow \operatorname{Eu} 3 \mathrm{~d}^{10} 4 \mathrm{f}^{n-1}+\mathrm{e}^{-}
$$

where "**" denotes an excited state, $n$ corresponds to the number of electrons filling the $4 \mathrm{f}$ level and is equal to $n=6$ or 7 for $\mathrm{Eu}^{2+}$ or $\mathrm{Eu}^{3+}$ states, respectively. The final states are hence equivalent to that which would result from a direct photoemission process of electrons out of the $4 \mathrm{f}$ levels. Due to the large photoionization cross section at $3 \mathrm{~d} \rightarrow 4 \mathrm{f}$ transition, the photoemission process from $4 \mathrm{f}$ states is highly favored, which explains high sensitivity to europium oxidation states in relation to $4 \mathrm{f}$ filling. The Eu $4 \mathrm{f}$ feature of the divalent europium appearing at $1128.8 \mathrm{eV}$ and made of a single contribution centered at $2.2 \mathrm{eV}$ of binding energy is very sensitive to the chemical environment of the europium ion and will be discussed later with the resonant $\mathrm{Eu}^{2+}$ uf signal measured at the $4 \mathrm{~d} \rightarrow$ ff transition. Finally, as the resonant electronic contribution coming from the $\mathrm{Eu}^{3+}$ and $\mathrm{Eu}^{2+}$ species are well separated, we determine the $\mathrm{Eu}^{2+}: \mathrm{Eu}^{3+}$ ratio from the integrated profiles of the resonant photoemission lines located at binding energies of $2.26 \mathrm{eV}$ and $7 \mathrm{eV}$. We calculate a relative weight of $15 \%$ in $\mathrm{Eu}^{2+}$ and $85 \%$ in $\mathrm{Eu}^{3+}$ for an inelastic electron mean free path of $21.76 \AA$.

RESPES spectra measured at the Eu $4 \mathrm{~d} \rightarrow 4 \mathrm{f}$ transition $\left(\mathrm{N}_{4,5}\right.$ absorption edge) are reported in Fig. 5. The X-ray absorption spectrum measured in total electron yield at the $4 \mathrm{~d} \rightarrow 4 \mathrm{f}$ transition is reported in the top panel. Compared to the $3 \mathrm{~d} \rightarrow$ 4f edge, the $4 \mathrm{~d} \rightarrow$ 4f XAS spectrum presents a singular broaden and asymmetric shape. The $4 \mathrm{~d} \rightarrow 4 \mathrm{f}$ transition is known to exhibit a response characteristic of a Fano-like shape component. This effect is related to localized excited states in the continuum. Clearly visible at the $4 \mathrm{~d} \rightarrow 4 \mathrm{f}$ transition edge, this behavior does not exist for $3 \mathrm{~d} \rightarrow 4 \mathrm{f}$ transitions presenting, as demonstrated above, a sharp resonant structure of atomic multiplet transitions with only Gaussian and Lorentzian broadenings of their discrete energy states.

As shown in a previous study, ${ }^{31}$ the signal coming from the $\mathrm{Eu}^{2+}$ and $\mathrm{Eu}^{3+}$ states are too close in energy at the $4 \mathrm{~d} \rightarrow 4 \mathrm{f}$ absorption edge to be clearly disentangled without any salt reference with the $\mathrm{Eu}^{2+} / \mathrm{Eu}^{3+}$ ratio. Indeed the single total yield NEXAFS spectrum is not sufficient to determine the ratio of $\mathrm{Eu}^{2+}$ and $\mathrm{Eu}^{3+}$ species. In order to quantify the amount of the two europium oxidation states, the RESPES spectrum has been measured.

The map reproducing the photoemission intensity measured when photon energy crosses $4 \mathrm{~d} \rightarrow 4 \mathrm{f}$ absorption edges is shown in the bottom panel of Fig. 5. The intensity distribution is very similar to the one observed at the $\mathbf{M}_{5}$ absorption edge. As seen in the $3 \mathrm{~d} \rightarrow$ 4f transition, two main resonant features are visible. The first one located at a binding energy of $2 \mathrm{eV}$ is normally induced by $\mathrm{Eu}^{2+}$ states and the second one located at around $7.5 \mathrm{eV}$ is commonly associated with $\mathrm{Eu}^{3+}$ states. ${ }^{29,31-33}$ Similar to

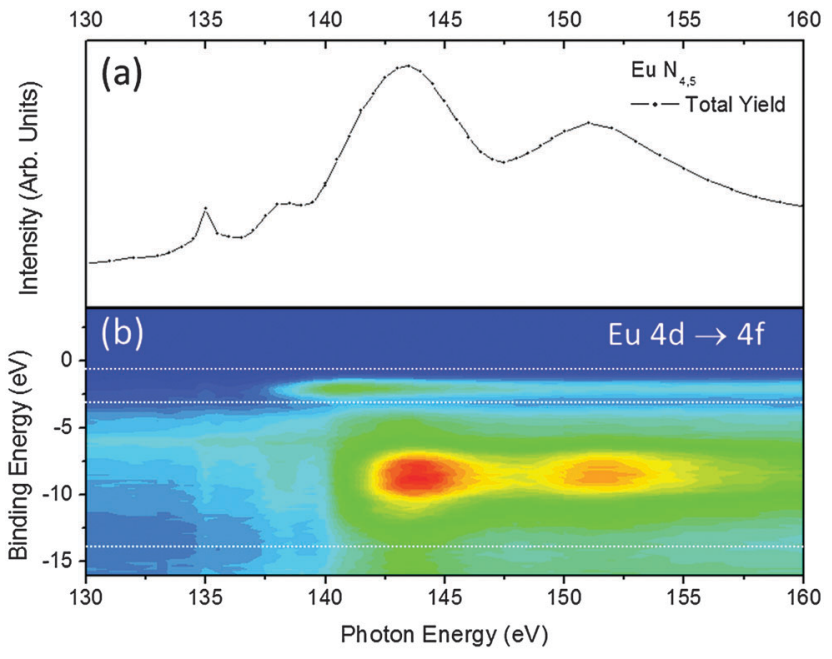

Fig. 5 (a) TEY X-ray absorption spectrum of the $\mathrm{EuCl}_{3}$ salt measured at the $4 \mathrm{~d} \rightarrow 4 \mathrm{f}$ absorption edge. (b) Resonant photoemission map in the binding energy range of $0-16 \mathrm{eV}$ measured while scanning the photon energy range of 130-160 eV across the Eu $\mathrm{N}_{4,5} 4 \mathrm{~d} \rightarrow 4 \mathrm{f}$ absorption edge. Dash lines delimit the area of localized resonant states.

$3 \mathrm{~d} \rightarrow 4 \mathrm{f}$ transitions, the resonant signals at $4 \mathrm{~d} \rightarrow 4 \mathrm{f}$ are described by a two-step excitation/de-excitation process according to the following equation:

$$
\text { Eu } 4 \mathrm{~d}^{10} 4 \mathrm{f}^{n}+h \nu \rightarrow\left(\operatorname{Eu~} 4 \mathrm{~d}^{9} 4 \mathrm{f}^{n+1}\right)^{*} \rightarrow \operatorname{Eu} 4 \mathrm{~d}^{10} 4 \mathrm{f}^{n-1}+\mathrm{e}^{-}
$$

with “*” designating an excited state, $n$ corresponding to the number of electrons filling the $4 \mathrm{f}$ level equal to, respectively, $n=6$ or 7 for $\mathrm{Eu}^{2+}$ or $\mathrm{Eu}^{3+}$ states. The observed final states are equivalent to those that would result from a direct photoemission process of electrons coming from $4 \mathrm{f}$ levels. Photoemission processes occurring from $4 \mathrm{f}$ states exhibit high sensitivity to europium oxidation states due to a large photoionization cross section at the $4 \mathrm{~d} \rightarrow 4 \mathrm{f}$ transition. At the $\mathrm{Eu}^{2+} 4 \mathrm{~d} \rightarrow 4 \mathrm{f}$ on-resonance, having a closer attention on the $\mathrm{Eu}^{2+}$ resonant feature, we notice that, as seen in the $\mathrm{Eu}^{2+} 3 \mathrm{~d} \rightarrow 4 \mathrm{f}$ on-resonance, the $\mathrm{Eu}^{2+} 4 \mathrm{f}$ contribution is composed of a single component located at a relatively high energy compared to the literature. ${ }^{20,27,47}$ In mixed valence transition compounds, the Eu $4 \mathrm{f}$ feature is made of two components corresponding to bulk and surface contributions located, respectively, at lower and higher binding energy and usually separated by less than $1 \mathrm{eV} .^{20,27,47}$ The high binding energy of the $\mathrm{Eu}^{2+}$ 4f component, that we measure, better fits with the surface state made of incomplete coordination europium at the origin of higher cohesive energy. ${ }^{43,47}$ Indeed, a high binding energy has already been observed in trivalent EuPt $_{5}$ presenting a divalent surface layer due to the surface valence transition. ${ }^{20}$ In addition, in that case only one component is observed corresponding to the divalent surface state. Actually, the Eu $4 \mathrm{f}$ energy position is intrinsically linked to the cohesive energy and the work function of the bonded element. Unfortunately, as we have only one $\mathrm{Eu}^{2+}$ contribution in the $4 \mathrm{f}$ spectrum, the screened core-hole model ${ }^{43}$ is not applicable to determine the cohesive energy. However, in pure ionic crystals, the cohesive energy is given by the interionic Coulomb interaction. 
This kind of interaction is of a long range that a very small fraction of defects or interactions with surrounding atoms dramatically affect the cohesive energy. In our case, $\mathrm{Eu}^{2+}$ species are embedded in the hydrated trivalent europium salt and act as a divalent europium ion in an incomplete coordination configuration. Two parameters then affect the cohesive energy, the presence of water molecules and of $\mathrm{Eu}^{2+}$ defects in the crystal. Indeed, the crystal structure shows that in the pure $\mathrm{EuCl}_{3} \cdot 6 \mathrm{H}_{2} \mathrm{O}$ salt, the europium ion is directly linked to $\mathrm{H}_{2} \mathrm{O}$ molecules ${ }^{49,50}$ and a recent study has shown that this interaction contributes to the broadening in optical transitions. ${ }^{6}$ In summary, the single Eu $4 \mathrm{f}$ contribution we measure combined with its high binding energy is in good agreement with a unique $\mathrm{Eu}^{2+}$ ground state. This is in agreement with a divalent europium ion in an incomplete coordination configuration homogeneously distributed throughout the crystal. From the resonant spectrum, shown in Fig. 6, we can figure out the peculiarities of $4 \mathrm{~d} \rightarrow 4 \mathrm{f}$ transitions. By integrating, at constant binding energy, the resonant photoemission intensity centered to the two resonant states, we obtain the profiles presented in the top panel of Fig. 6 where they are compared to the spectral shape measured in total electron yield. The resonant lines are composed of mainly one asymmetric component centered at $139 \mathrm{eV}$ in photon energy corresponding to divalent europium and a second one composed of two asymmetric features at 143 and $152 \mathrm{eV}$ ascribed to the trivalent europium species.

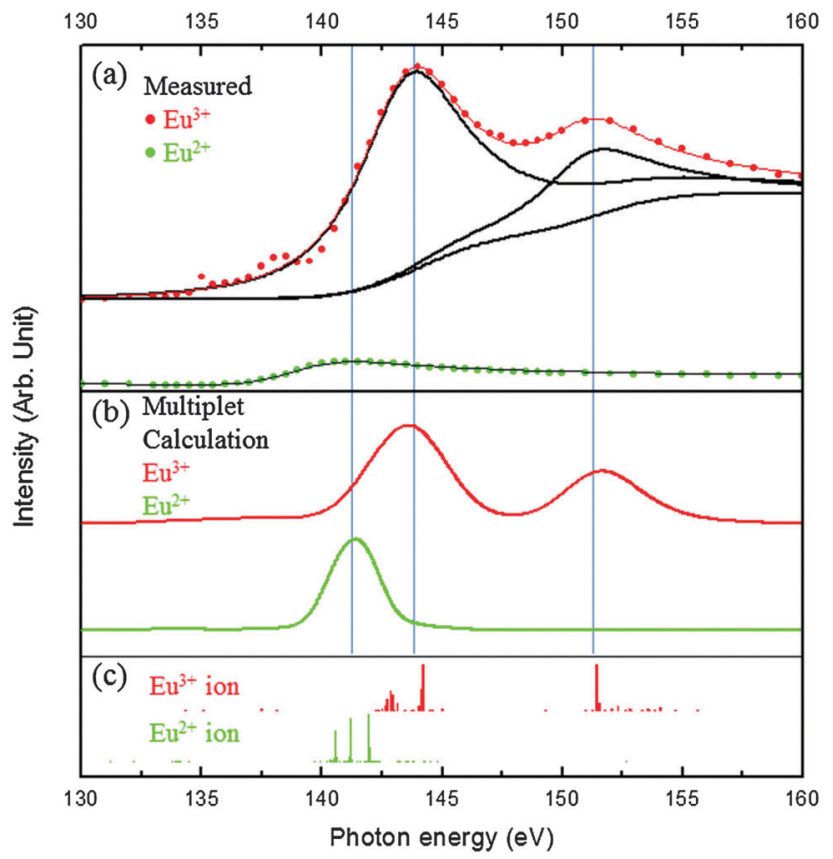

Fig. 6 (a) $N_{4,5}$ absorption edges measured in partial electron yield integrating around the resonant lines located at $2 \mathrm{eV}$ and $8.3 \mathrm{eV}$ binding energy with Fano like profile calculations. The red curve is decomposed in two asymmetric Fano profiles and a continuum step lineshape background while the green curve is only composed of one asymmetric Fano profile. (b) $\mathrm{Eu}^{2+}$ and $\mathrm{Eu}^{3+}$ calculated XAS spectra at the $4 \mathrm{~d} \rightarrow 4 \mathrm{f}$ absorption edge using the CTM4XAS 5.23 program (in green and red, respectively). The

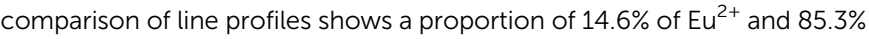
of $\mathrm{Eu}^{3+}$ species. (c) Representation of discrete energy states of intraatomic multiplet excitation of $\mathrm{Eu}^{2+}$ and $\mathrm{Eu}^{3+}$ ions.
The partial yield lineshapes can be compared with those computed from the atomic multiplet model shown in the central panel of Fig. 6. In the bottom panel of Fig. 6c, we present the discrete energy states of intra-atomic multiplet $4 \mathrm{~d} \rightarrow 4 \mathrm{f}$ excitations of $\mathrm{Eu}^{2+}$ and $\mathrm{Eu}^{3+}$ ions. The broadening of discrete energy states leads to calculated XAS spectra shown in Fig. 6c. Atomic multiplet calculations at the $4 \mathrm{~d} \rightarrow 4 \mathrm{f}$ transition are performed with a lifetime broadening of the core-hole of $0.2 \mathrm{eV}$, calculated with a natural width of $\mathrm{Eu} 4 \mathrm{~d}$ states $^{42}$ and an instrumental resolution broadening of $0.7 \mathrm{eV}$ and $1.5 \mathrm{eV}$ for divalent and trivalent europium, respectively, as found in the $\mathrm{Eu} 4 \mathrm{~d}$ core level spectra. We obtain good agreement in terms of position and balance between different components corresponding to divalent and trivalent europium species. However, this model does not describe the broadening and the asymmetric shape of different components. The asymmetry of the $4 \mathrm{~d} \rightarrow 4 \mathrm{f}$ resonant photoemission feature can be fitted using a Fano profile. ${ }^{51}$ In agreement with the Fano model, the interference of a discrete autoionized state with a continuum results in a typical asymmetric peak in excitation spectra. A giant resonant asymmetric profile verifies the following expression:

$$
\begin{gathered}
I=I_{0} \frac{(q+\varepsilon)^{2}}{1+q^{2}}+I_{\mathrm{b}} \\
\varepsilon=\frac{E_{\mathrm{Phot}}-E_{\mathrm{r}}}{\Gamma / 2}
\end{gathered}
$$

where $E_{\mathrm{phot}}$ is the incident photon energy, $E_{\mathrm{r}}$ the energy at resonance, and $I_{0}$ and $I_{\mathrm{b}}$ are the intensity contributions to transitions from continuum toward states that interact (i.e. resonant contribution) and do not interact (i.e. nonresonant part of the signal) with the discrete autoionized state, respectively. $|q|$ is the Fano asymmetry parameter related to the lineshape and $\Gamma$ indicates the spectral width of the autoionized state. In Fig. 6, we present an asymmetric Fano profile for the divalent and trivalent europium contributions.

The $\mathrm{Eu}^{2+}$ component matches well with a unique asymmetric component verifying eqn (1). $\mathrm{Eu}^{3+}$ is a sum of two asymmetric components superimposed onto a continuum step lineshape background. ${ }^{52}$ We take the width of the steps equal to the linewidth of the $\mathrm{Eu}^{3+}$ resonant components. According to eqn (1), the calculated energy resonances $E_{\mathrm{r}}$ at the Eu $4 \mathrm{~d} \rightarrow 4 \mathrm{f}$ transition for $\mathrm{Eu}^{2+}$ and $\mathrm{Eu}^{3+}$ main components are equal to $139 \mathrm{eV}$ for $\mathrm{Eu}^{2+}$ and $143 \mathrm{eV}$ for the first component of $\mathrm{Eu}^{3+}$ species and $152 \mathrm{eV}$ for the second one, respectively. The linewidth of the $\mathrm{Eu}^{2+}$ state is about $6.6 \mathrm{eV}$ while the two components of the $\mathrm{Eu}^{3+}$ states present a lower natural linewidth of $5.5 \mathrm{eV}$. The linewidths of the autoionized state are somewhat broader compared with the theoretical energy states spread (tick figure) combined with the experimental enlargement determined in Eu $4 \mathrm{~d}$ core level photoemission spectra (Fig. 2). The asymmetric parameter $|q|$ is lower in the case of $\mathrm{Eu}^{2+}$ states $\left(\left|q_{\mathrm{Eu}^{2+}}\right|=1.5\right)$ compared to the $\mathrm{Eu}^{3+}$ states $\left(\left|q_{\mathrm{Eu}^{3+}}\right|=12.5\right)$. This means that $\mathrm{Eu}^{2+}$ states exhibit stronger interactions between the discrete state and the states of the continuum. ${ }^{53}$ These interactions are also visible in core level photoemission as discussed previously in 
Fig. 2 where $\mathrm{Eu}^{3+}$ core level components exhibit a standard Voigt doublet taking into account the spin orbit splitting of $4 \mathrm{~d}_{3 / 2}$ and $4 \mathrm{~d}_{5 / 2}$ states whereas $\mathrm{Eu}^{2+}$ contribution presents an asymmetric shape due to d-hole and the ground state coupling. The results are in good agreement with Fano models, the $\mathrm{Eu}^{2+}$ state can be understood as an interaction of one discrete state with states of a continuum while $\mathrm{Eu}^{3+}$ exhibits a behavior characteristic of the interaction of one discrete state with states of two or more continua.

Indeed, the interaction of one discrete state with states of two or more continua results in an asymmetric peak, as for the interaction between one state and one continuum, superposed on a smooth background. ${ }^{51,54}$ The origin of $4 \mathrm{~d} \rightarrow 4 \mathrm{f}$ asymmetric shape resonance has been abundantly discussed for years. ${ }^{55-61}$ However, since Eu is known to be a highly spin polarized system, new calculations based on the dynamic electron correlation decomposing in spin-up and spin-down states and the contribution of $4 \mathrm{~d}$ electrons have shown that a sharp resonance of $4 \mathrm{~d}$ spin-up electrons (quasi-bound state) interaction with a broad resonance of $4 \mathrm{~d}$ spin-down electrons (viewed as a continuum) is responsible for the $4 \mathrm{~d}$ giant resonance exhibiting a strong asymmetric Fano profile. ${ }^{62}$ This explanation is also consistent with the multiplet structure observed for the $3 \mathrm{~d} \rightarrow 4 \mathrm{f}$ transition: while the $4 \mathrm{~d}$ level exhibits a low spin orbit splitting of about $5 \mathrm{eV},{ }^{63}$ the spin-up and spin-down electrons are close enough to interact together so as to lead to giant resonance with the Fano shape profile, 3d levels present higher spin orbit splitting beyond $30 \mathrm{eV}^{63}$ avoiding spin-up and spin-down electron interactions and leading to resonance in good agreement with atomic multiplet calculations.

Removing the background from different line profiles (Fig. 6a), we integrate the area of each resonant line profile at $4 \mathrm{~d} \rightarrow$ ff transitions associated with the $\mathrm{Eu}^{3+}$ and $\mathrm{Eu}^{2+}$ species to determine their relative weight. The comparison of the two areas gives a percentage of $85 \%$ of $\mathrm{Eu}^{3+}$ and $15 \%$ of $\mathrm{Eu}^{2+}$ species for an inelastic electron mean free path of $5.72 \AA$. This result confirms the amounts found at $3 \mathrm{~d} \rightarrow 4 \mathrm{f}$ transitions corresponding to a higher inelastic mean free path of $21.76 \AA$. We show that the amount of $\mathrm{Eu}^{2+}$ species remains constant as a function of the sample depth. The $\mathrm{Eu}^{2+}$ species are homogeneously distributed into the film.

The measurement of $4 \mathrm{~d} \rightarrow 4 \mathrm{f}$ resonant photoemission spectra corroborates the results obtained at the $3 \mathrm{~d} \rightarrow 4 \mathrm{f}$ absorption edge. In both cases we clearly identify the signature of divalent and trivalent europium ions. In opposition to other

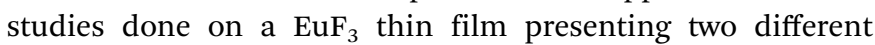
spectroscopic signatures at $3 \mathrm{~d} \rightarrow 4 \mathrm{f}$ and $4 \mathrm{~d} \rightarrow 4 \mathrm{f}$ transitions, ${ }^{33}$ our sample does not exhibit any probed depth dependence. The relative weight of $\mathrm{Eu}^{2+}$ and $\mathrm{Eu}^{3+}$ electronic features measured at $\mathrm{M}_{4,5}$ and $\mathrm{N}_{4,5}$ absorption edges represent 15 and 85\%, respectively, which correspond to a $\mathrm{EuCl}_{2.85}$ mixed valence salt close to the $\mathrm{EuCl}_{2.85 \pm 0.05}$ composition determined by chemical dosing. This result confirms a homogeneous surface and bulk repartition of the two $\mathrm{Eu}^{2+}$ and $\mathrm{Eu}^{3+}$ oxidation states in the sample with no surface-induced valence transition to the divalent state. ${ }^{18}$

\section{Conclusions}

By measuring the Eu $4 \mathrm{~d}$ core level spectra as a function of the photon energy, the spectral signature of $\mathrm{Eu}^{2+}$ and $\mathrm{Eu}^{3+}$ states of the hydrated $\mathrm{EuCl}_{2.85}$ salt has been evidenced. When compared to the trivalent europium, the divalent europium presents a complex feature due to $4 \mathrm{~d} \rightarrow 4 \mathrm{f}$ interactions. Both contributions exhibit a broad line shape. This feature is attributed to defects, due to the coexistence of divalent and trivalent ions in the crystal and to the interaction with surrounding water molecules. In particular, the spectroscopic signature of divalent europium shows that the ion lies in an incomplete coordination configuration. The amount of divalent and trivalent ions remains constant as a function of the sample depth. These results are in good agreement with a homogeneous distribution of the divalent europium into the film. Core level photoemission spectroscopy is a very efficient and accurate technique to determine a spatial concentration profile by comparing the evolution in shape of the core level spectra by varying the probing depth. The calculated composition of the salt presents a slight deviation compared to the composition determined by the chemical analysis. The uncertainty originates from the difficulty to disentangle the signals coming from the $\mathrm{Eu}(\mathrm{II})$ and $\mathrm{Eu}(\mathrm{III})$ species deeply entangled and superimposed on a complex background.

By comparison, a more precise quantification of two species is obtained from resonant photoemission experiments in the valence band region performed at $3 \mathrm{~d} \rightarrow 4 \mathrm{f}$ and $4 \mathrm{~d} \rightarrow 4 \mathrm{f}$ absorption edges. We have shown that mixed-valence hydrated europium chloride thin film exhibits two well separated contributions corresponding to $\mathrm{Eu}^{2+}$ and $\mathrm{Eu}^{3+}$ spectroscopic signatures. The multiplet calculation, in good agreement with $3 \mathrm{~d} \rightarrow 4 \mathrm{f}$ photoabsorption, allowed us to clearly identify the $\mathrm{Eu}^{2+}$ and $\mathrm{Eu}^{3+}$ contributions as measured in partial electron yield. The spectra obtained by integrating the resonant intensity located at around $2 \mathrm{eV}$ and $8 \mathrm{eV}$ binding energies are well understood and identified by calculations and corresponding XAS total electron yield spectral shapes. All the observed contributions can be assigned to $\mathrm{Eu}^{2+}$ and $\mathrm{Eu}^{3+}$ valence states, respectively.

Similarly, the resonant photoemission spectra at $4 \mathrm{~d} \rightarrow 4 \mathrm{f}$ transition exhibits two main de-excitation pathways related to divalent and trivalent europium ions. At lower photon energy the giant resonance shape is obviously characteristic of Fanolike line shapes. This effect related to transitions from localized excited states to continuum is most pronounced for $4 \mathrm{~d} \rightarrow 4 \mathrm{f}$ transition while it is not observable for $3 \mathrm{~d} \rightarrow 4 \mathrm{f}$ absorption spectra which can be reproduced by atomic multiplet calculation, provided that the broadenings due to experimental resolution and core-hole lifetime of atomic multiplet discrete energy states are taken into account. The integration of $\mathrm{Eu}^{2+}$ and $\mathrm{Eu}^{3+}$ resonant states across the $\mathrm{M}_{4,5}$ and $\mathrm{N}_{4,5}$ absorption edges gives profile lines with relative weights close to 15 and $85 \%$ (respectively) at both edges. The relative weight in electronic behavior is in perfect agreement with the chemical composition of the salt.

From the combination of the core level photoemission spectra and resonant photoemission spectra, we have emphasized the robustness and accuracy in chemical depth profiling 
of ionic solids. Applied to hydrated mixed valence $\mathrm{EuCl}_{2.85}$, we have demonstrated the homogeneous distribution of the divalent europium in the crystal leading to a bulk $n$ doping of the salt.

These spectroscopic techniques exhibit a very high sensitivity to chemical species down to 0.1 atomic percent. ${ }^{64}$ They provide an efficient alternative to conventional chemical analysis to detect impurities at low concentration.

\section{Notes and references}

1 L. M. Moreno, Europium: Compounds, Production and Applications, Nova Science Publishers, Inc., Hauppauge NY, 2011.

2 H. de Riedmatten, M. Afzelius, M. U. Staudt, C. Simon and N. Gisin, Nature, 2008, 456, 773-777.

3 E. Fraval, M. J. Sellars and J. J. Longdell, Phys. Rev. Lett., 2005, 95, 030506.

4 A. L. Alexander, J. J. Longdell, M. J. Sellars and N. B. Manson, Phys. Rev. Lett., 2006, 96, 043602.

5 J. J. Longdell, E. Fraval, M. J. Sellars and N. B. Manson, Phys. Rev. Lett., 2005, 95, 063601.

6 R. L. Ahlefeldt, A. Smith and M. J. Sellars, Phys. Rev. B: Condens. Matter Mater. Phys., 2009, 80, 205106.

7 J. C. G. Bunzli and C. Piguet, Chem. Soc. Rev., 2005, 34, 1048-1077.

8 A. Matsko, D. Strekalov and L. Maleki, Opt. Express, 2005, 13, 2210-2223.

9 M. Fleischhauer and M. Lukin, Phys. Rev. Lett., 2000, 84, 5094-5097.

10 M. Fleischhauer, A. Imamoglu and J. Marangos, Rev. Mod. Phys., 2005, 77, 633-673.

11 W. Dehorrocks and M. Albin, Prog. Inorg. Chem., 1983, 31, 1-104.

12 M. G. Silly, S. Blanchandin, F. Sirotti, F. Lux, S. Chevreux, G. Lemercier and F. Charra, J. Phys. Chem. C, 2013, 117, 9766-9771.

13 S. Kasap, P. Capper and H. Tuller, Springer Handbook of Electronic and Photonic Materials, Editon edn, Springer, USA, 2007, pp. 213-228.

14 M. R. Oliver, J. O. Dimmock, A. L. McWhorter and T. B. Reed, Phys. Rev. B: Condens. Matter Mater. Phys., 1972, 5, 1078-1098.

15 A. Schmehl, V. Vaithyanathan, A. Herrnberger, S. Thiel, C. Richter, M. Liberati, T. Heeg, M. Rockerath, L. F. Kourkoutis, S. Muhlbauer, P. Boni, D. A. Muller, Y. Barash, J. Schubert, Y. Idzerda, J. Mannhart and D. G. Schlom, Nat. Mater., 2007, 6, 882-887.

16 R. P. Panguluri, T. S. Santos, E. Negusse, J. Dvorak, Y. Idzerda, J. S. Moodera and B. Nadgorny, Phys. Rev. B: Condens. Matter Mater. Phys., 2008, 78, 125307.

17 S. G. Altendorf, A. Efimenko, V. Oliana, H. Kierspel, A. D. Rata and L. H. Tjeng, Phys. Rev. B: Condens. Matter Mater. Phys., 2011, 84, 155442.

18 G. K. Wertheim and G. Crecelius, Phys. Rev. Lett., 1978, 40, 813-816.
19 B. Johansson, Phys. Rev. B: Condens. Matter Mater. Phys., 1979, 19, 6615-6619.

20 E. J. Cho, S. J. Oh, S. Suga, T. Suzuki and T. Kasuya, J. Electron Spectrosc. Relat. Phenom., 1996, 77, 173-181.

21 E. J. Cho and S. J. Oh, Phys. Rev. B: Condens. Matter Mater. Phys., 1999, 59, R15613-R15616.

22 W. D. Schneider, C. Laubschat, I. Nowik and G. Kaindl, Phys. Rev. B: Condens. Matter Mater. Phys., 1981, 24, 5422-5425.

23 M. P. Seah and W. A. Dench, Surf. Interface Anal., 1979, 1, 2-11.

24 C. D. Wagner, L. E. Davis and W. M. Riggs, Surf. Interface Anal., 1980, 2, 53-55.

25 C. Laubschat, E. Weschke, G. Kalkowski and G. Kaindl, Phys. Scr., 1990, 41, 124-129.

26 C. Felser, J. Kohler, A. Simon, O. Jepsen, G. Svensson, S. Cramm and W. Eberhardt, Phys. Rev. B: Condens. Matter Mater. Phys., 1998, 57, 1510-1514.

27 K. Yamamoto, K. Horiba, M. Taguchi, M. Matsunami, N. Kamakura, A. Chainani, Y. Takata, K. Mimura, M. Shiga, H. Wada, Y. Senba, H. Ohashi and S. Shin, Phys. Rev. B: Condens. Matter Mater. Phys., 2005, 72, 161101.

28 S. Banik, A. Bendounan, A. Thamizhavel, A. Arya, P. Risterucci, F. Sirotti, A. K. Sinha, S. K. Dhar and S. K. Deb, Phys. Rev. B: Condens. Matter Mater. Phys., 2012, 86, 085134.

29 B. A. Orlowski, P. Dziawa, B. Kowalski, I. Kowalik, M. Pietrzyk, V. Osinniy, T. Story, S. Mickievicius and R. Jonhson, Appl. Surf. Sci., 2006, 252, 5379-5383.

30 B. A. Orlowski, B. J. Kowalski, M. Pietrzyk and R. Buczko, Acta Phys. Pol., A, 2008, 114, S103-S114.

31 W. D. Schneider, C. Laubschat, G. Kalkowski, J. Haase and A. Puschmann, Phys. Rev. B: Condens. Matter Mater. Phys., 1983, 28, 2017-2022.

32 J. Szade, W. Burian, Z. Celinski, T. O’Keevan, M. Zangrando, F. Bondino and E. Magnano, Surf. Sci., 2005, 580, 163-166.

33 J. Szade, W. Burian, M. Zangrando, F. Bondino, E. Magnano, S. Widuch and Z. Celinski, Surf. Sci., 2008, 602, 1525-1531. 34 M. Zhuravleva, S. Friedrich and C. L. Melcher, Opt. Mater., 2014, 36, 670-674.

35 F. Polack, M. Silly, C. Chauvet, B. Lagarde, N. Bergeard, M. Izquierdo, O. Chubar, D. Krizmancic, M. Ribbens, J. P. Duval, C. Basset, S. Kubsky and F. Sirotti, 10th International Conference on Synchrotron Radiation Instrumentation, Melbourne, AUSTRALIA. AIP Conf. Proc., 2010, 1234, 185.

36 N. Bergeard, M. G. Silly, D. Krizmancic, C. Chauvet, M. Guzzo, J. P. Ricaud, M. Izquierdo, L. Stebel, P. Pittana, R. Sergo, G. Cautero, G. Dufour, F. Rochet and F. Sirotti, J. Synchrotron Radiat., 2011, 18, 245-250.

37 E. Stavitski and F. M. F. de Groot, Micron, 2010, 41, 687-694.

38 R. Cowan, The Theory of Atomic Structure and Spectra, University of California Press, Berkeley, 1981.

39 S. Tanuma, C. J. Powell and D. R. Penn, Surf. Interface Anal., 1994, 21, 165-176.

40 D. L. Perry, Handbook of Inorganic Compounds, Second edn, CRC Press, 2011.

41 P. A. Bruhwiler, O. Karis and N. Martensson, Rev. Mod. Phys., 2002, 74, 703-740. 
42 C. Gerth, K. Godehusen, M. Richter, P. Zimmermann, J. Schulz, P. Wernet, B. Sonntag, A. G. Kochur and I. D. Petrov, Phys. Rev. A: At., Mol., Opt. Phys., 2000, 61, 022713.

43 B. Johansson and N. Martensson, Phys. Rev. B: Condens. Matter Mater. Phys., 1980, 21, 4427-4457.

44 C. Caspers, M. Muller, A. X. Gray, A. M. Kaiser, A. Gloskovskii, C. S. Fadley, W. Drube and C. M. Schneider, Phys. Status Solidi RRL, 2011, 5, 441-443.

45 B. T. Thole, G. Vanderlaan, J. C. Fuggle, G. A. Sawatzky, R. C. Karnatak and J. M. Esteva, Phys. Rev. B: Condens. Matter Mater. Phys., 1985, 32, 5107-5118.

46 J. B. Goedkoop, B. T. Thole, G. Vanderlaan, G. A. Sawatzky, F. M. F. Degroot and J. C. Fuggle, Phys. Rev. B: Condens. Matter Mater. Phys., 1988, 37, 2086-2093.

47 N. Martensson, B. Reihl, W. D. Schneider, V. Murgai, L. C. Gupta and R. D. Parks, Phys. Rev. B: Condens. Matter Mater. Phys., 1982, 25, 1446-1448.

48 S. Iacobucci, F. Sirotti, M. Sacchi and G. Stefani, J. Electron Spectrosc. Relat. Phenom., 2002, 123, 397-401.

49 D. L. Kepert, J. M. Patrick and A. H. White, Aust. J. Chem., 1983, 36, 477-482.

50 N. K. Belskii and Y. T. Struchkov, Sov. Phys. Cryst., 1965, 10, 15.

51 U. Fano, Phys. Rev., 1961, 124, 1866.

52 J. Stöhr, NEXAFS Spectroscopy, XV edn, Springer, 1992.

53 R. Kumar, Indian J. Phys., 2013, 87, 49-52.
54 U. Fano and J. W. Cooper, Phys. Rev., 1965, 137, 1364.

55 J. W. Cooper, Phys. Rev. Lett., 1964, 13, 762.

56 K. T. Cheng and C. F. Fischer, Phys. Rev. A: At., Mol., Opt. Phys., 1983, 28, 2811-2819.

57 K. T. Cheng and W. R. Johnson, Phys. Rev. A: At., Mol., Opt. Phys., 1983, 28, 2820-2828.

58 U. Becker, H. G. Kerkhoff, D. W. Lindle, P. H. Kobrin, T. A. Ferrett, P. A. Heimann, C. M. Truesdale and D. A. Shirley, Phys. Rev. A: At., Mol., Opt. Phys., 1986, 34, 2858-2864.

59 M. Richter, M. Meyer, M. Pahler, T. Prescher, E. Vonraven, B. Sonntag and H. E. Wetzel, Phys. Rev. A: At., Mol., Opt. Phys., 1989, 39, 5666-5675.

60 M. Richter, M. Meyer, M. Pahler, T. Prescher, E. Vonraven, B. Sonntag and H. E. Wetzel, Phys. Rev. A: At., Mol., Opt. Phys., 1989, 40, 7007-7019.

61 C. Pan, S. L. Carter and H. P. Kelly, Phys. Rev. A: At., Mol., Opt. Phys., 1991, 43, 1290-1300.

62 X. M. Tong, D. Kato, T. Watanabe and S. Ohtani, J. Phys. B: At., Mol. Opt. Phys., 2000, 33, 717-725.

63 D. Attwood, E. Gullikson, M. Howells, K.-J. Kim, J. Kirz, J. Kortright, I. Lindau, Y. Liu, P. Pianetta, A. Robinson, J. Scofield, J. Underwood, G. Williams and H. Winick, $X-R A Y$ DATA BOOKLET, Center for X-ray Optics and Advanced Light Source, Lawrence Berkeley National Laboratory, 2009.

64 K. Kiss, Problem Solving with Microbeam Analysis, Elsevier, 1988, vol. 7, ch. 9, pp. 148-153. 